\title{
Addressing Security and Human Rights Challenges in Complex Environments
}

\author{
Alan BRYDEN* and Lucía HERNÁNDEZ**
}

Keywords: business, complex environment, human rights, private actors, security

\section{INTRODUCTION}

Among the challenges faced by businesses operating in complex environments, security issues can be particularly difficult to address. One reason for this is that a company's responsibility to respect human rights extends not only to its own operations and people but to the individuals, communities, and states in which they operate. Indeed, this is the key principle underpinning international initiatives such as the United Nations Guiding Principles on Business and Human Rights (UNGPs) and the Voluntary Principles on Security and Human Rights (VPs). The twin imperatives of 'inward'- and 'outward'-looking security and human rights commitments may not always be easily reconcilable.

The International Committee of the Red Cross (ICRC) and the Geneva Centre for the Democratic Control of Armed Forces (DCAF) are committed to promoting effective multi-stakeholder approaches to security and human rights challenges. Both organizations are official Observers to the VPs. This engagement has translated into a strategic partnership, supported by the Swiss Federal Department of Foreign Affairs, seeking to maximize the opportunities offered by the VPs through working together with companies, states, and civil society organizations to help address security and human rights challenges. The DCAF-ICRC partnership has developed the Addressing Security and Human Rights Challenges in Complex Environments Toolkit ${ }^{1}$ and an accompanying Knowledge Hub. ${ }^{2}$ These products are intended to provide practical guidance that is freely available to all stakeholders with an interest in promoting security and human rights with regard to businesses operating in complex environments.

\footnotetext{
* Head of the Public-Private Partnerships Division at DCAF.

** Project Coordinator at DCAF.

1 The Toolkit can be accessed at http://www.securityhumanrightshub.org/content/toolkit (accessed 30 July 2015).

2 The Knowledge Hub can be accessed at http://www.securityhumanrightshub.org (accessed 30 July 2015).
} 
This article highlights insights and approaches emerging from the DCAF-ICRC partnership. Following the logic of the project, it is intended to promote much-needed discussion and experience sharing across stakeholder groups and knowledge communities. The article begins by summarizing important challenges, as expressed by companies at headquarters and operational levels. It then identifies lessons learned and relevant good practices. Finally, it considers progress made to date and assesses the challenges that remain in this field.

\section{Security and Human Rights Challenges}

Although security and human rights challenges are always context specific, companies share many common concerns. These challenges fall under three broad categories which align with key stakeholder groups that companies engage with when managing the security and safety of their operations: host governments, public security forces, and private security providers. $^{3}$

\section{A. Host Governments}

States have the duty to protect human rights, promote the rule of law, and provide security within their territory. To effectively address security and human rights challenges, a close relationship between the host government and the company is therefore essential. However, developing predictable relations with host governments is one of the main challenges faced by companies. It can be extremely difficult to identify entry points within the host government and to co-ordinate with the different national and local authorities and agencies working on these issues. Moreover, raising human rights or other security-related concerns with host governments can be a sensitive topic. Even when political will exists to address these issues, lack of capacity and resources may prevent counterparts from effectively delivering on agreements made with companies.

Despite the frequent gap between the letter of security arrangements agreed at the national level and the reality of their implementation on the ground, many companies invest considerable efforts in establishing a written agreement, joint protocol, or Memorandum of Understanding (MoU) with the host government. While few companies succeed in establishing a comprehensive MoU addressing all relevant aspects of engagement, this process may permit the establishment of concrete agreements on specific areas of concern such as training or equipment transfers. ${ }^{4}$

\section{B. Public Security Forces}

The relationship with public security forces is often perceived as posing the most significant potential security and human rights risk to companies operating in complex environments. A major challenge that companies can face when working with public

\footnotetext{
3 As reflected by the three chapters of the Addressing Security and Human Rights Challenges in Complex Environments Toolkit.

4 Since little guidance on how to conduct this process is publicly available, DCAF and the ICRC are working with IPIECA, the global oil and gas industry association for environmental and social issues, to jointly develop templates and other practical tools that can support responsible security practices by companies engaging with host governments.
} 
security forces relates to poor vetting and training programmes, lack of appropriate equipment and limited accountability for their actions. While it is the host government's responsibility to provide the required resources, training and equipment, and to ensure effective oversight and accountability of national security forces, the reality can differ considerably from this ideal scenario. In these situations, some companies try to minimise the extent of their relations with public security, by relying as much as possible on private security providers or in-house security. However, some level of interaction is not only unavoidable but also necessary, since private security cannot and should not assume law enforcement functions.

As part of a programme for its Rumaila oilfield operations in Southern Iraq, British Petroleum has been working with the Iraqi Government to develop a human rights training assistance programme. ${ }^{5}$ The programme, targeted at the Iraqi Oil Police Force $(\mathrm{OPF})$, includes a number of innovative elements. Significantly, the programme has adopted a train-the-trainer approach, in order to promote ownership and sustainability by developing a cadre of OPF trainers with the skills and knowledge to train their colleagues. The training has a long term perspective: a three-year training cycle of foundation, intermediate, and advanced courses and is mentored throughout by training consultants with professional experience in the public security forces, and human rights issues, and local language skills. The training also includes an important evaluation component in order to measure its impact on the performance of OPF police officers and to identify further training needs.

\section{Private Security Providers}

Other than the issues common to public or private security (e.g. vetting, training, equipment), one specific challenge concerns the supply and demand for private security in complex environments. In some contexts, neither international nor local providers meet the requisite standards, presenting the company with tricky choices and the need to weigh risks. Working as a private security guard is often one of the lowest paying forms of employment, despite the risks and responsibilities that such a job entails. As in any other sector, when salaries are low and working conditions are poor, individuals are likely to lack qualifications and motivation, which increases the risk of poor performance and security incidents. 'Moonlighting' is another common challenge. Public security personnel may take on a second job as private security guards, creating confusion over roles and responsibilities, in particular with regard to the appropriate use of force.

These challenges are compounded by weak regulation at the national level, contributing to a lack of transparency and accountability of the industry. In 2014, the first ever meeting of states from francophone and lusophone Africa on private security regulation highlighted a number of such challenges. To give one example, in Senegal private security guards are entitled to certain terms and conditions, including an agreed minimum wage. However, in practice this is often ignored with individuals often given short-term contracts with salaries (when they are paid) that are significantly lower than required. Indeed, it is reported that the majority of complaints against companies come

5 'Case Study: Engagement with public security forces in Rumaila - Iraq', http://www.securityhumanrightshub.org/ content/case-studies (accessed 30 July 2015). 
from their own employees. ${ }^{6}$ These practices undoubtedly contribute to poor performance and increase the risk of human rights abuses.

\section{Good Practices And Lessons LeARned}

The issues presented above only provide a snapshot of the array of security and human rights challenges related to corporate operations in complex environments. However, given their prevalence, efforts to tackle these challenges can make a significant contribution to improving corporate security management. Working directly with stakeholders at headquarters and field level, in a process of shared learning and development, DCAF and the ICRC are developing practical, implementation-orientated guidance and tools that seek to help companies manage the security of operations in a way that respects human rights and international humanitarian law. The approach is deliberately non-prescriptive and simply provides a point of departure for the development of context- and company-specific practices. However, looking at the project as a whole, a number of good practices are generally applicable. These include: ensuring internal coherence in policies and processes; engaging with communities from the outset; and collaborating widely with relevant stakeholders.

- Ensuring internal coherence in policies and processes throughout the company is crucial to enable the implementation of good practices. This requires regular communication and co-ordination between relevant departments (e.g. those dealing with operations, legal issues, government relations, security, corporate social responsibility, and community relations) to avoid duplication, contradictory messages to external stakeholders, or the development of unsuitable policies and processes. Companies may require support in order to ensure the development of appropriate policies and processes. In Peru, Socios Peru, a specialist nongovernmental organization, is working with extractives companies to develop new approaches to address security and human rights challenges faced by the companies. Socios Peru uses the VPs as a baseline for dialogue but then translates this to reflect local realities and develop solutions that respond to local needs. This approach benefits from the multi-stakeholder dynamics created by the national VPs working group-which includes six VPs member companies and eight governments.

- Good community relations is possibly the best risk reduction strategy a company could have. This means engaging with communities as early as possible on security arrangements, showing respect for the local culture, sharing information about potential risks and impacts, ensuring all groups are adequately represented in consultations, and establishing locally appropriate processes to jointly address challenges. This requires time, flexibility, and openness. While companycommunity relations will not guarantee the absence of disagreements or even some tensions, it establishes a framework of mutual trust and dialogue which is the essence of a prevention-based approach to security and human rights. In 2013,

6 DCAF, Rapport de la Conférence régionale en Afrique francophone et lusophone, Geneva, 2015, 23. 
Kosmos Energy started Cameroon's first ever onshore oil project. ${ }^{7}$ This meant that the security and human rights challenges were new to all the stakeholders involved. Accordingly, in order to address potential risks linked to its security arrangements, community engagement was prioritized in developing the company's approach to training public security forces. Through a partnership with the NGO Fund for Peace, a process of close engagement with community leaders and other prominent community representatives was developed in order to provide the basis for the design of human rights training for the military unit responsible for extractive operations security. The insights gained from communities ensured a focus on the actual challenges faced on the ground. It also enabled the mainstreaming of values shared between the armed forces and communities-family, honour, respect, and human security - in the design and roll out of the training.

- Working with host and home governments, companies, industry associations, civil society organizations and other relevant stakeholders is likely to offer the most effective route to addressing sensitive security and human rights issues. A multistakeholder approach not only provides perspective, but also enables collective learning and the pooling of resources while lending legitimacy to any measures taken. Multi-stakeholder forums such as the VPs Working Groups established in some countries, provide exactly this kind of platform for discussion around security challenges and how to address them. Since 2012, regular multi-stakeholder security meetings have been held in Lubumbashi to discuss challenges and share good practices around extractives operations in the Katanga province of the Democratic Republic of the Congo. ${ }^{8}$ Participation is open to all extractives companies in the area, local public institutions, public security forces, private security companies, human rights organizations, and the United Nations Organization Stabilization Mission in the Democratic Republic of the Congo (MONUSCO). This multistakeholder forum pools knowledge to address day-to-day security challenges. At the same time, the meetings have an important confidence-building dimension across companies, public authorities, and civil society organizations.

\section{Conclusion}

A willingness to exchange frankly across stakeholder groups on what works and what does not is an essential pre-requisite to learning lessons and identifying better ways of doing business. This is the real value-add of multi-stakeholder initiatives such as the VPs. Drawing on this experience, two general observations seem particularly relevant.

First, it is essential to bridge gaps between policy and practice. Principles and policy documents establish a baseline of acceptable norms and standards. They do not provide the level of granularity to allow field and headquarters personnel to establish specific policies and management processes or to develop operational guidance that can be

7 'Case Study: Human Rights Training - Cameroon', http://www.securityhumanrightshub.org/content/case-studies (accessed 30 July 2015).

8 'Case Study: Monthly Security and Human Rights Meetings in Lubumbashi', http://www.securityhumanrightshub. org/content/case-studies (accessed 30 July 2015). 
applied on the ground. Such guidelines need to be translated into tools that are adaptable to the realities and constraints faced by individual companies and the contexts in which they operate. There is a careful balance to be struck to ensure that guidance is neither so general as to be practically useless nor so specific as to be irrelevant beyond a particular place and time. Moreover, the process is as important as the result. Good practices that emerge as the result of a process of co-development with companies and other stakeholders are much more likely to be implemented.

Second, stovepiping of actors and approaches remains a reality, resulting in lost knowledge and unrealized synergies. The business and human rights and security sector reform (SSR) communities provide a case in point. On the one hand, the business and human rights community can tap into both the operational experience and the political and governance dynamics that underpin the SSR approach to supporting effective, well managed, and democratically-accountable security sectors. On the other hand, the SSR community is only just beginning to think about how private actors fit within SSR approaches and can profit greatly from the significant advances made in the business and human rights field. However, interactions to date between the two knowledge communities are few and ad hoc. In short, there is much to be gained from mutual exchange and sufficiently blended interests to make such cooperation feasible.

The DCAF-ICRC partnership was established to facilitate understanding of challenges on the ground, develop practical guidance and tools, and promote knowledge sharing. These goals can only be achieved through close co-operation and engagement with different stakeholders at headquarters and in the field. We hope that this article may contribute to building bridges and establishing partnerships that can help to address security and human rights challenges in complex environments. ${ }^{9}$

9 If you are interested in engaging with the DCAF-ICRC project, please contact PPPs@dcaf.ch. 